\title{
Evaluation of Surgical Procedures that Affect the Hemodynamics Using the FloTrac System in Esophageal Cancer Patients
}

\author{
MAKOTO SOHDA ${ }^{1}$, KENGO KURIYAMA $^{1}$, YUJI KUMAKURA ${ }^{1}$, TOMONORI YOSHIDA ${ }^{1}$, \\ HIROAKI HONJYO ${ }^{1}$, MAKOTO SAKAI ${ }^{1}$, TATSUYA MIYAZAKI ${ }^{1}$, MASAFUMI KANEMOTO ${ }^{2}$, \\ MASARU TOBE ${ }^{2}$, HIROSHI HINOHARA ${ }^{2}$, SHIGERU SAITO ${ }^{2}$ and HIROYUKI KUWANO ${ }^{1}$ \\ ${ }^{I}$ Department of General Surgical Science, Gunma University Graduate School of Medicine, Maebashi, Japan; \\ ${ }^{2}$ Department of Anesthesiology, Gunma University Graduate School of Medicine, Maebashi, Japan
}

\begin{abstract}
Background/Aim: Esophagectomy is more invasive compared to other gastrointestinal surgery types. Perioperative circulatory management is important to avoid postoperative heart complications. The FloTrac sensor along with the Vigileo monitor is a minimally invasive haemodynamic monitoring device. Here, we examined different surgical procedures affecting hemodynamics using the FloTrac system in esophageal cancer patients following esophagectomy. Patients and Methods: Thirty-one patients undergoing postoperative monitoring with the FloTrac sensor/Vigileo monitor system following esophagectomy were included. Evaluation of cardiac index (CI) and stroke volume variation (SVV) measurements were performed by analyzing the number of aberrant values. We evaluated the correlation between the number of aberrant values of CI, SVV and surgical methods of esophagectomy and perioperative factors. Results: There was no significant correlation between the number of aberrant values of CI, SVV and operative approach or fields of lymphadenectomy. Regarding the reconstruction route following esophagectomy, there was a significant correlation between the number of aberrant values of $C I, S V V$ and retrosternal route compared with other routes. There was a significant correlation between the number of aberrant values of SVV and preoperative heart complication. Conclusion: Hemodynamic stability management using FloTrac/Vigileo system following esophagectomy is
\end{abstract}

This article is freely accessible online.

Correspondence to: Makoto Sohda, Department of General Surgical Science, Gunma University Graduate School of Medicine, 3-39-22 Showa-machi, Maebashi, Gunma 371-8511, Japan. Tel: +81 272208224, Fax: +81 272208230, e-mail: msohda@gunma-u.ac.jp

Key Words: FloTrac sensor, retrosternal route, hemodynamic, esophageal cancer, esophagectomy. useful for safe performance of postoperative management of esophageal cancer patients.

Improvement of surgical techniques and perioperative management of esophagectomy are among the factors decreasing morbidity and mortality in esophageal cancer patients. Perioperative circulatory management is especially important, because esophagectomy, which involves two- or three-phase procedures, such as abdominal, chest and/or neck incisions, is more invasive than other types of gastrointestinal surgery. Postoperative hypovolemic hypotension following esophagectomy can cause a shift of extracellular fluid from the central to peripheral compartments (1). Multiple minimally invasive hemodynamic monitoring devices, including pulse pressure/stroke volume variation (SVV), esophageal Doppler, and extravascular lung water measurement, have been evaluated for the optimization of the perioperative fluid therapy (1). The FloTrac/Vigileo system (Edwards Lifescience, Tokyo, Japan) is a less invasive hemodynamic monitoring device compared to those used for thermodilution assessment. This device can continuously monitor cardiac output (CO), as well as stroke volume and SVV through a peripheral arterial pressure line. Kobayashi et al., have reported SVV is an accurate predictor of intravascular hypovolemia and a useful indicator for assessing the appropriateness and timing of applying fluid to improve circulatory stability during the perioperative period following esophagectomy (2).

The non-invasive FloTrac sensor does not require for calibrated $\mathrm{CO}$ monitoring. The advantage of the system is that it can offer very valuable information easily, as it is directly connected to the arterial pressure waveform. Moreover, the FloTrac system is useful for regulating the infusion volume. Isosu et al., have suggested that SVV measurement is useful for estimating the optional volume for fluid supplementation and may prevent excessive fluid 
infusion during surgical procedures, such as laparoscopic adrenalectomy in patients with pheochromocytoma (3). The recent report also reports the usefulness of fluid adjustment using Flotrac in many cancer surgery (4-6).

In a report on esophageal cancer, Ishihara et al., have reported that none of the tested variables (SVV, pulse pressure variation, central venous pressure, intrathoracic blood volume and initial distribution volume of glucose) can accurately predict fluid responsiveness following abdominothoracic esophagectomy, even for the near future (7). However, Kobayashi et al., have concluded that SVV is an accurate predictor of intravascular hypovolemia and useful for assessing the appropriateness and timing of applying fluid for improving circulatory stability during the perioperative period following esophagectomy (2).

Surgery alone or in combination with chemotherapy or chemoradiation is the mainstay in treatment of localized esophageal adenocarcinoma and one of several options for esophageal squamous cell cancer, which may also be radically treated with definitive chemoradiotherapy or radiotherapy alone. Surgical resection is a common treatment for patients with resectable esophageal cancer, however, the most appropriate surgical approach is subject to considerable debate, and major controversies surround the issue of the operative approach, such as video-assisted surgery versus open surgery, selection of conduit, methods of reconstruction and curability of resection and so on. For the first time, we examined some of the surgical procedures that affect the hemodynamics using the FloTrac system in esophageal cancer patients following esophagectomy.

\section{Patients and Methods}

Patient characteristics and operation procedure. Between April 2013 and May 2014, 31 patients (25 males, 6 females) with a mean age of $66.6 \pm 8.3$ (range, $45-81$ ) years underwent postoperative monitoring with the FloTrac sensor/Vigileo monitor system following curative surgery for esophageal cancer at Gunma University Hospital, Japan. The FloTrac sensor/Vigileo monitor system was used in the intensive care unit (ICU). Each patient gave written informed consent before esophagectomy. Preoperative examinations regarding operable tolerance included heart, respiratory, renal and hepatic functions, and all enrolled patients passed the preoperative examination. Preoperative echocardiographic measurements of the left ventricular ejection fraction were normal in all patients. Patients with pacemakers, a history of cardiac arrhythmia, severe peripheral vascular disease, cardiac support or persisting mitral or aortic dysfunction after surgery were excluded.

Patient characteristics are shown in Table I. In total, 20 patients underwent radical surgery for esophageal cancer performed using a right thoracoabdominal approach, whereas 11 patients were operated using video-assisted thoracoscopic esophagectomy. Twenty-four patients underwent esophagectomy with an extended three-field lymphadenectomy, and the remaining 7 patients underwent two-field lymphadenectomy. Moreover, reconstruction was performed using a gastric tube as a conduit in all cases except

Table I. Patient characteristics.

Patient characteristics $(n=31)$

\section{Gender}

Age (years; mean \pm SD)

Esophagectomy

Thoracoscopic surgery

Traditional surgery

Reconstruction Route

Postmediastional route

Retrostinal route

Antethoracic route

Anastomosis position

Cervix

High intrathoracic

Fields of lymphadenectomy

2 fields

3 fields

Operation time

(minutes; mean $\pm \mathrm{SD}$ )

M: Male; F: female; SD: standard deviation.

for a single case of right colon interposition. With regards to the reconstruction route and anastomosis position, 27 patients were operated via the postmediastinal route $(87.1 \%)$, with 21 cases of neck esophagogastrostomy and 6 cases of high intrathoracic esophagogastrostomy. Three patients were selected for the retrosternal route with neck esophagogastrostomy, and only one patient was operated through the antethoracic route with colon interposition, as described above.

Postoperative care and assessment of haemodynamic parameters in $I C U$. Prior to the induction of anesthesia, a $20 \mathrm{G}$ catheter was inserted in the left radial artery and was connected to the FloTrac/Vigileo monitor (Edwards Lifescience, Irvine, CA, USA). The FloTrac/Vigileo system monitored: i) CO, ii) CI, iii) stroke volume (SV), iv) stroke volume index (SVI) and v) SVV. We retrospectively calculated and analysed CI and SVV values in ICU. $\mathrm{CI}$ is indicated as: $\mathrm{CO}(1 / \mathrm{min}) /$ patient body surface. SVV $(\%)$, similarly, equals: (maximum SV-minimum SV)/mean SV, where maximum and minimum $\mathrm{SV}$ are the mean values of the four extreme values of SV during a period of 30 seconds, and the mean $\mathrm{SV}$ is the average value for this time period.

Postoperatively, all patients were kept intubated and ventilated overnight in ICU, and if their clinical condition was satisfactory, they were extubated in the next morning. The 21-bedded ICU was available to all esophageal cancer patients and was staffed by anaesthetists and experienced nurses. Routinely, 1,000 $\mathrm{ml}$ of 5\% $(12.5 \mathrm{~g} / 250 \mathrm{ml})$ albumin (CSL Behring) was immediately instilled in each ICU patient to provide circulatory stabilization. Blood transfusion was avoided unless serum haemoglobin persistently fell below $9 \mathrm{~g} / \mathrm{dl}$ or the clinical situation was otherwise deteriorated. A continuous intravenous injection of $0.2 \mathrm{mg} / \mathrm{kg}$ per hour of sivelestat sodium hydrate (ONO Pharmacol, Osaka, Japan) was also administered at ICU.

FloTrac sensor data were collected from the patients admitted to ICU, and the length of their stay was recorded. CI and SVV were 

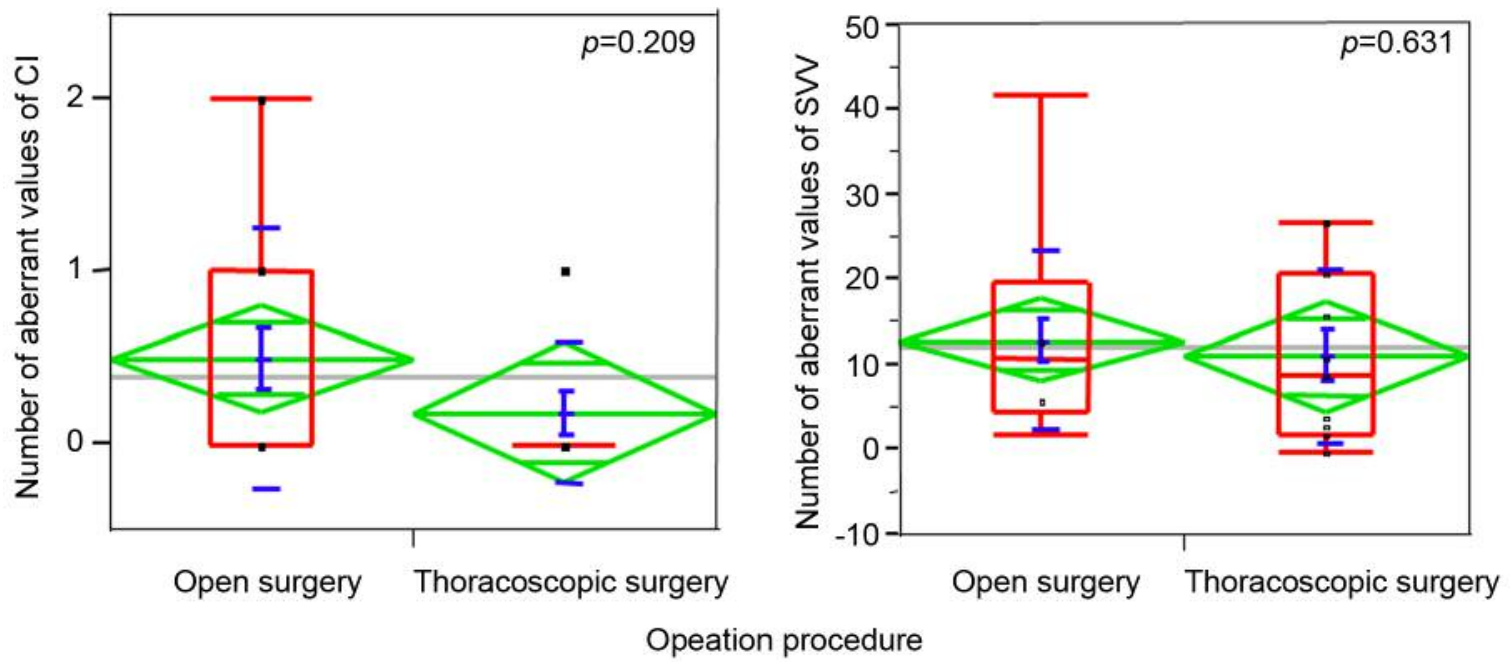

Figure 1. Correlation between the number of aberrant values of cardiac index (CI), stroke volume variation (SVV) and operative approach (thoracoscopic versus open surgery). The green diamonds represent the sample mean and its confidence interval. The red box plot shows a concise representation of the distribution of values, and blue line shows confidence interval and its mean value.
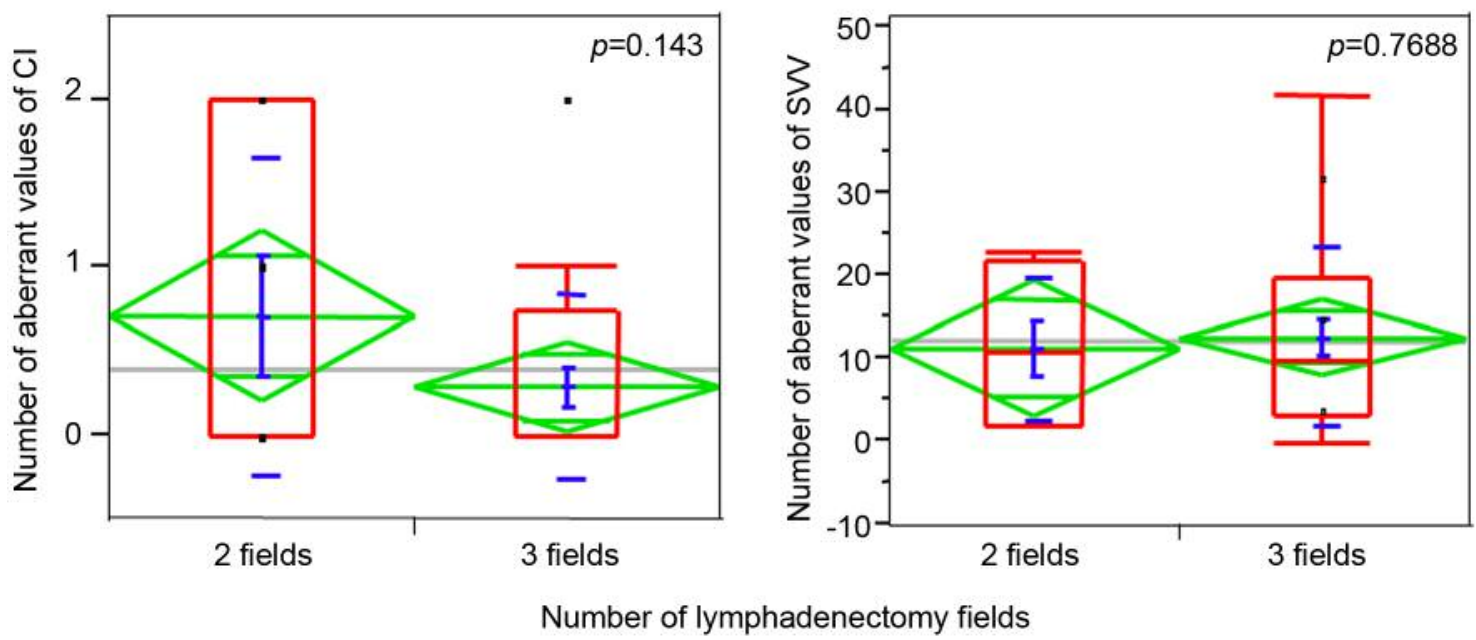

Figure 2. Correlation between the number of aberrant values of cardiac index (CI), stroke volume variation (SVV) and number of lymphadenectomy fields.

evaluated by recording the number of aberrant values. In this study, CI values $<2.2$ and SVV values $>10$ were considered aberrant as per previous reports. We evaluated the correlation between the number of aberrant CI and SSV values along with surgical methods of esophagectomy or other perioperative factors.

Statistical analysis. The relationship between the number of aberrant CI and SVV values and surgical methods of esophagectomy and other perioperative factors was analysed using Fisher's ANOVA. Probability $(p)$ values $<0.05$ were considered as indicative of statistically significant differences.

\section{Results}

There was no significant correlation between the number of aberrant values of CI, SVV and sex or age (cutoff: 70 years old). With regards to the operation procedure, there was no significant correlation between the number of aberrant values of CI, SVV and operative approach (thoracoscopic versus traditional open surgery) (Figure 1). Moreover, there was no significant correlation between the number of aberrant values of CI, SVV and the number of lymphadenectomy fields (2 

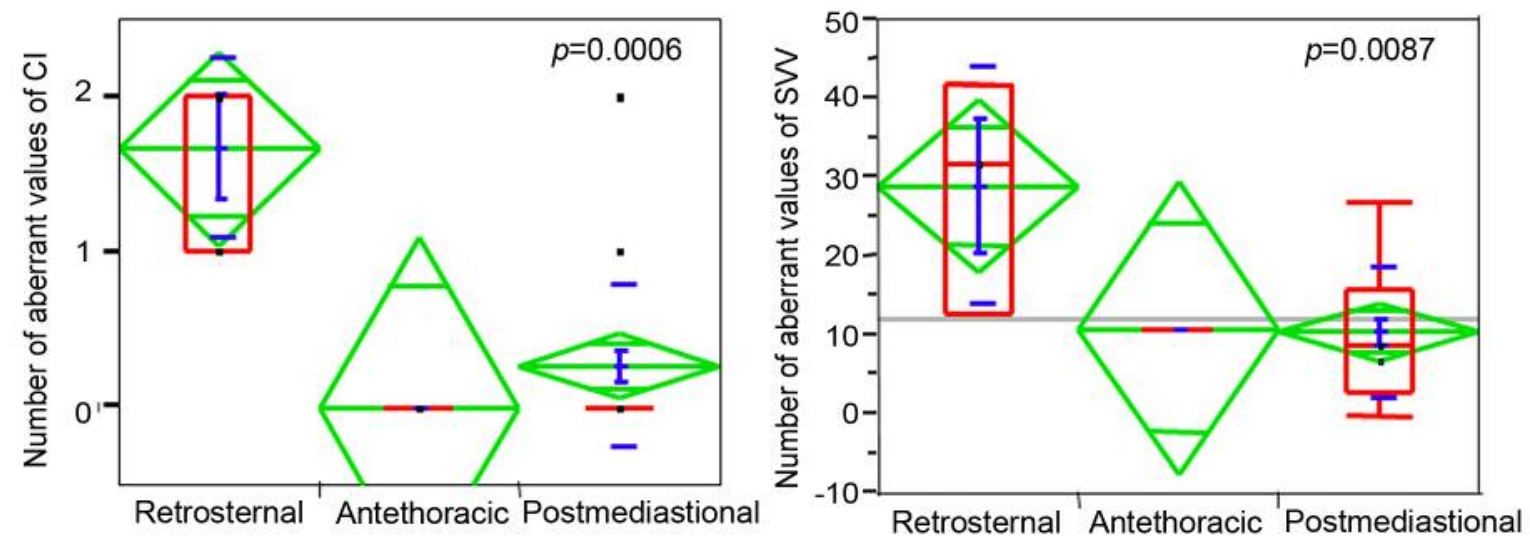

Reconstruction route

Figure 3. Correlation between number of aberrant values of cardiac index (CI), stroke volume variation (SVV)and retrosternal route compared to other routes.

fields versus 3 fields) (Figure 2). Regarding the reconstruction route following esophagectomy, there was a significant correlation between the number of aberrant values of CI, SVV and retrosternal route compared to other routes (Figure 3 ). The retrosternal route had a significantly negative effect on hemodynamics in both $\mathrm{CI}$ and SVV compared to the antethoracic and postmediastinal route.

Concerning the perioperative data, we assessed the operation time, the intraoperative blood transfusion, the intraoperative bleeding and the balance of intraoperative infusions. There was no significant correlation between the number of aberrant values of CI, SVV and these perioperative factors.

Moreover, we analysed the correlation between the number of aberrant values of CI, SVV and preoperative heart complications, such as hypertension, atrial fibrillation or angina. There was a significant correlation between the number of aberrant values of SVV and preoperative heart complications (Figure 4). This result shows that esophageal cancer patients with heart complications tend to be hypovolemic during the postoperative period.

\section{Discussion}

The FloTrac/Vigileo system device uses arterial pressure waveform analysis to calculate $\mathrm{SV}$ and $\mathrm{CO}$, without a requirement for calibration against an independent $\mathrm{CO}$ measurement. Therefore, it provides a method to determine the hemodynamics status, changes in the clinical course and response to therapeutic intervention in patients with arterial catheters in place (8).

To the best of our knowledge, there is no study available comparing CI, SVV and surgical procedures or perioperative

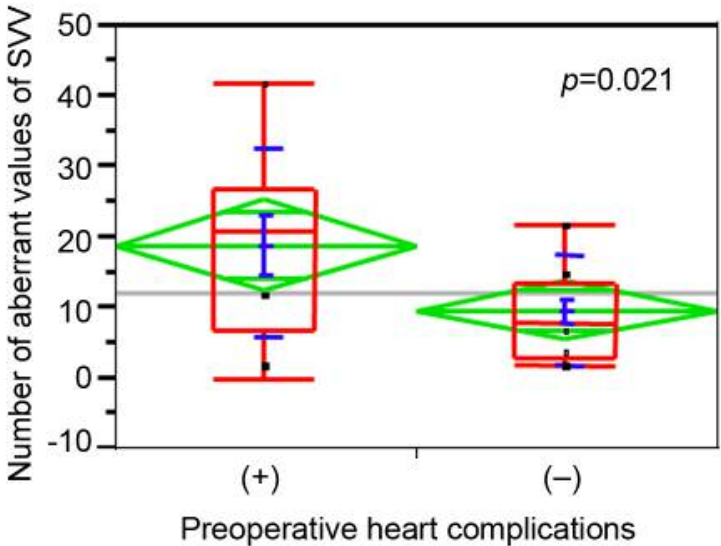

Figure 4. Correlation between the number of aberrant values of stroke volume variation (SVV) and preoperative heart complications.

factors affecting hemodynamics using the FloTrac system in esophageal cancer patients.

Using the FloTrac/Vigileo haemodynamic monitor, the comparison between CI and SVV measurements with surgical procedure data in patients undergoing esophagectomy showed that there was no significant correlation between the number of aberrant values of CI, SVV and the operative approach (thoracoscopic versus traditional open surgery).

VATS esophagectomy has several advantages over open esophagectomy, such as better cosmesis, reduced tissue trauma, reduced pain, reduced postoperative inflammatory response and lower morbidity rates (9-11), although massive active bleeding and bronchial injury have also been reported as major intraoperative complications of VATS esophagectomy (12). At first, we anticipated that thoracoscopic surgery was 
less invasive compared to traditional open surgery with regards to the patient's haemodynamic stability. However, there was no significant correlation between the number of aberrant values of CI, SVV and the type of operative approach. We have usually performed muscle-sparing thoracotomy for esophagectomy in esophageal cancer patients as a less invasive procedure. The current result shows that our traditional, less invasive open esophagectomy (muscle-sparing thoracotomy) is a less invasive operative approach compared to thoracoscopic surgery in the aspect of hemodynamic stability for esophageal cancer. Moreover, with regards to the post-esophagectomy reconstruction route, we showed there is a significant correlation between the number of aberrant values of CI, SVV and retrosternal route compared to other routes. Reconstructive options following esophagectomy can be categorized according to: i) the choice of conduit, such as stomach, colon or jejunum, ii) specifics of conduit construction, such as whole stomach or gastric tube left or right colon, or need for microvascular augmentation, iii) location of anastomosis, such as cervical or thoracic, iv) route of reconstruction, such as postmediastinal, retrosternal or antethoracic, and v) necessity of gastric drainage procedure, such as pyloplasty, pylomyotomy or no drainage (13-15).

The retrosternal and posterior mediastinal routes are the most commonly used gastric tube routes for reconstruction following esophagectomy when cervical esophagogastric anastomosis is performed (16-18). Bartels et al., have reported that reconstruction in the posterior mediastinum is associated with significantly fewer days spent in the intensive therapy unit, fewer cardiopulmonary complications and lower mortality (16). They have concluded that the posterior mediastinal route is strongly recommended for patients with cardiopulmonary risk factors. Urschel et al., have reported comparable results in a meta-analysis of randomized controlled trials (19). However, a recent report by Zheng et al., has shown that the reconstruction route is not associated with any significant variability in the extent of cardiac or pulmonary complications (20). Our data here show that the retrosternal route potentially influences the haemodynamic stability measured by the FloTrac/Vigileo system. However, there were no apparent clinical symptoms at the time of the aberrant values. Moreover, proponents of the retrosternal route of conduit transposition place the gastric conduit outside the tumour bed to avoid interference of the mediastinal tumour recurrence with alimentary tract function (21). In addition, postoperative irradiation can be performed on the tumour bed to lessen the chances of tumour recurrence without damaging the conduit. We think that reconstruction through the retrosternal route following esophagectomy is a very important option for locally advanced esophageal cancer. However, it is not preferable to reconstruct using the retrosternal route in esophageal cancer patients with cardiac complications.
Regarding the perioperative data, there was no significant correlation between the number of aberrant values of CI, SVV and perioperative factors. Transfusion management is important for sustaining haemodynamic stability in patients following esophagectomy. These data witness the excellent management of anaesthetists and nurses in ICU.

Wakabayashi et al., have shown that SVV is an accurate predictor of intravascular hypovolemia and a useful indicator for assessing the appropriateness and timing of applying fluid for improving circulatory stability during a giant hemangioma resection (22). Our data show that esophageal cancer patients with heart complications markedly tended to present with aberrant SSV values during the postoperative period. This result does not necessarily suggest a potential hypovolemia following esophagectomy in patients with heart complications. Instead, this indicates that adequate transfusion management during anaesthesia is required to avoid postoperative heart complication.

To the best of our knowledge, this is the first report using the FloTrac system to demonstrate whether surgical procedures affect the hemodynamics in esophageal cancer patients following esophagectomy. It is important to obtain a thorough comprehension of the haemodynamic stability following esophagectomy, as this is an invasive operation and individual responses to surgical stress from this type of surgery vary and are difficult to predict, bearing a high risk of perioperative cardiorespiratory instability. Therefore, haemodynamic stability management using the FloTrac/Vigileo system following esophagectomy is useful for the safe performance of the postoperative management of esophageal cancer patients. The limitation of this study is the small number of cases, and it is, therefore, necessary to accumulate data on additional cases and from other health institutions.

\section{Conflicts of Interest}

The Authors have no conflicts of interest to declare.

\section{Authors' Contributions}

All Authors took part in the design of the study, contributed to data collection, and participated in writing the manuscript.

\section{Acknowledgements}

The Authors thank Ms. Yuka Matsui for her technical assistance during submission.

\section{References}

1 Chau EH and Slinger P: Perioperative fluid management for pulmonary resection surgery and esophagectomy. Semin Cardiothorac Vasc Anesth 18: 36-44, 2014. PMID: 23719773. DOI: $10.1177 / 1089253213491014$ 
2 Kobayashi M, Koh M, Irinoda T, Meguro E, Hayakawa Y and Takagane A: Stroke volume variation as a predictor of intravascular volume depression and possible hypotension during the early postoperative period after esophagectomy. Ann Surg Oncol 16: 1371-1377, 2009. PMID: 19219508. DOI: 10.1245/ s10434-008-0139-0

3 Isosu T, Obara S, Ohashi S, Hosono A, Nakano Y, Imaizumi T, Mogami M, Iida $\mathrm{H}$ and Murakawa M: Examination of the usefulness of non-invasive stroke volume variation monitoring for adjusting fluid supplementation during laparoscopic adrenalectomy in patients with pheochromocytoma. Fukushima J Med Sci 58: 78-81, 2012. PMID: 22790896.

4 Zeng K, Li Y, Liang M, Gao Y, Cai H and Lin C: The influence of goal-directed fluid therapy on the prognosis of elderly patients with hypertension and gastric cancer surgery. Drug Des Devel Ther 29: 2113-2119, 2014. PMID: 25378913. DOI: 10.2147/ DDDT.S66724

5 Giustiniano E, Procopio F, Ruggieri N, Grimaldi S, Torzilli G and Raimondi F: Impact of the FloTrac/VigileoTM Monitoring on Intraoperative Fluid Management and Outcome after Liver Resection. Dig Surg 35: 435-441, 2018. PMID: 29073613. DOI: 10.1159/000481406

6 Gottin L, Martini A, Menestrina N, Schweiger V, Malleo G, Donadello K and Polati E: Perioperative fluid administration in pancreatic surgery: A comparison of three regimens. J Gastrointest Surg, 2019. PMID: 30945088. DOI: 10.1007/s116 05-019-04166-4

7 Ishihara H, Hashiba E, Okawa H, Saito J, Kasai T and Tsubo T: Neither dynamic, static, nor volumetric variables can accurately predict fluid responsiveness early after abdominothoracic esophagectomy. Perioper Med (Lond) 2: 3, 2013. PMID: 24472615. DOI: 10.1186/2047-0525-2-3

8 Argueta E, Berdine G, Pena C and Nugent KM: FloTrac ${ }^{\circledR}$ Monitoring System: What are its uses in critically ill medical patients? Am J Med Sci 349: 352-356, 2015. PMID: 25584624. DOI: 10.1097/MAJ0000000000000393

9 Biere SSA, Cuesta MA and Van Del Peet DL: Minimally invasive versus open esophagectomy for cancer: a systematic review and meta-analysis. Minerva Chir 64: 121-133, 2009. PMID: 19365313.

10 Sgourakis G, Gockel I, Radtke A, Musholt TJ, Timm S, Rink A, Tsiamis A, Karaliotas C and Lang H: Minimally invasive versus open esophagectomy: metaanalysis of outcomes. Dig Dis Sci 55: 3031-3040, 2010. PMID: 20186484. DOI: 10.1007/s10620-0101153-1

11 Nagpal K, Ahmed K, Vats A, Yakoub D, James D, Ashrafian H, Darzi A, Moorthy $\mathrm{K}$ and Athanasiou T: Is minimally invasive surgery beneficial in the management of esophageal cancer? A meta-analysis. Surg Endosc 24: 1621-1629, 2010. PMID: 201 08155. DOI: $10.1007 / \mathrm{s} 00464-009-0822-7$
12 Shichinose T, Hirano $\mathrm{S}$ and Kondo S: Video-assisted esophagectomy for esophageal cancer. Surg Today 38: 206-213, 2008. PMID: 18306993. DOI: 10.1007/s00595-007-3606-5

13 Murakami M, Sugiyama A, Ikegami T, Aruga H, Matsushita K, Ishida K, Maruta F, Ikeno T, Shimizu F and Kawasaki S: Additional microvascular anastomosis in reconstruction after total esophagectomy for cervical esophageal carcinoma. Am J Surg 178: 263-266, 1999. PMID: 10527451.

14 Horváth OP, Lukács L and Cseke L: Complications following esophageal surgery. Recent Results Cancer Res 155: 161-173, 2000. PMID: 10693250

15 Urschel JD: Does the interponat affect outcome after esophagectomy for cancer? Dis Esophagus 14: 124-130, 2001. PMID: 11553222. DOI: 10.1046/j.1442-2050.2001.00169.x

16 Bartels H, Thorban S and Siewert JR: Anterior versus posterior reconstruction after transhiatal oesophagectomy: a randomized controlled trial. Br J Surg 80: 1141-1144, 1993. PMID: 8402115.

17 van Lanschot JJ, Hop WC, Voormolen MH, van Deelen RA, Blomjous JG and Tilanus HW: Quality of palliation and possible benefit of extra-anatomic reconstruction in recurrent dysphagia after resection of carcinoma of the esophagus. J Am Coll Surg 179: 705-713, 1994. PMID: 7524974.

18 van Lanschot JJ, van Blankenstein M, Oei HY and Tilanus HW: Randomized comparison of prevertebral and retrosternal gastric tube reconstruction after resection of oesophageal carcinoma. $\mathrm{Br}$ J Surg 86: 102-108, 1999. PMID: 10027371. DOI: 10.1046/ j.1365-2168.1999.00981.x

19 Urschel JD, Urschel DM, Miller JD, Bennett WF and Young JE: A meta-analysis of andomized controlled trials of route of reconstruction after esophagectomy for cancer. Am J Surg 182: 470-475, 2001. PMID: 11754853.

20 Zheng YZ, Dai SQ, Li W, Cao X, Wang X, Fu JH, Lin P, Zhang LJ, Lu B and Wang JY: Comparison between different reconstruction routes in esophageal squamous cell carcinoma. World J Gastroenterol 18: 5616-5621, 2012. PMID: 23112556. DOI: $10.3748 /$ wjg.v18.i39.5616

21 Gawad KA, Busch C and Izbicki JR: The route of reconstruction following esophagectomy. Zentralbl Chir 126: 2-8, 2001. PMID: 11819162 .

22 Wakabayashi S, Yamaguchi K, Kugimiya $\mathrm{T}$ and Inada E: Successful anesthetic management for resection of a giant hepatic hemangioma with Kasabach-Merritt syndrome using FloTrac system. Masui 60: 1326-1330, 2011. PMID: 22175175.

Received April 25, 2019

Revised June 10, 2019

Accepted June 20, 2019 